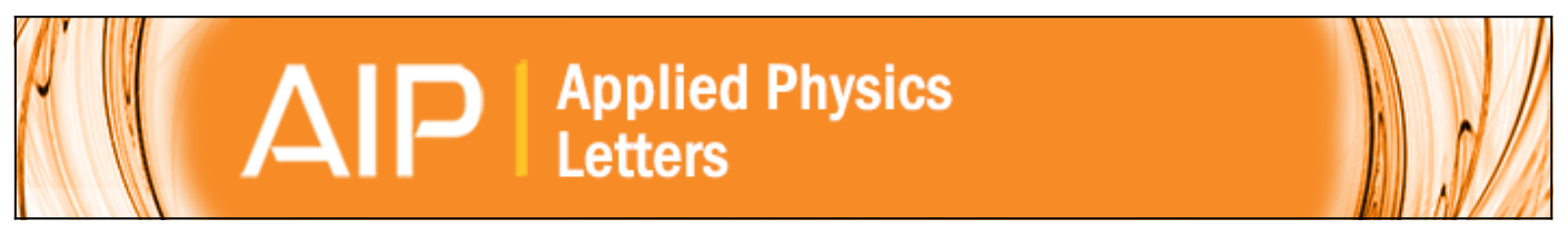

\title{
All-optical switching in optically induced nonlinear waveguide couplers
}

Falko Diebel, Daniel Leykam, Martin Boguslawski, Patrick Rose, Cornelia Denz, and Anton S. Desyatnikov

Citation: Applied Physics Letters 104, 261111 (2014); doi: 10.1063/1.4886414

View online: http://dx.doi.org/10.1063/1.4886414

View Table of Contents: http://scitation.aip.org/content/aip/journal/apl/104/26?ver=pdfcov

Published by the AIP Publishing

\section{Articles you may be interested in}

Publisher's Note: "All-optical switching in optically induced nonlinear waveguide couplers" [Appl. Phys. Lett. 104, $261111(2014)]$

Appl. Phys. Lett. 105, 099901 (2014); 10.1063/1.4894538

High-contrast all optical bistable switching in coupled nonlinear photonic crystal microcavities

Appl. Phys. Lett. 96, 131114 (2010); 10.1063/1.3378812

Integrated all-optical switch in a cross-waveguide geometry

Appl. Phys. Lett. 88, 171104 (2006); 10.1063/1.2197931

All-optical switching and beam steering in tunable waveguide arrays

Appl. Phys. Lett. 86, 051112 (2005); 10.1063/1.1857071

All-optical beam deflection and switching in strontium-barium-niobate waveguides

Appl. Phys. Lett. 72, 1960 (1998); 10.1063/1.121317

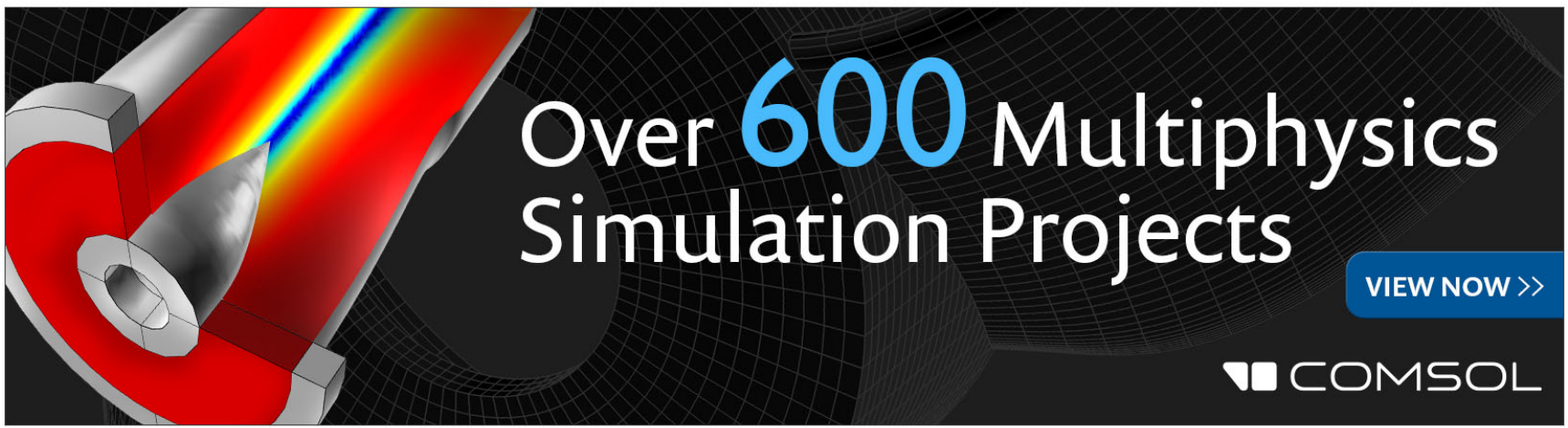




\title{
All-optical switching in optically induced nonlinear waveguide couplers
}

\author{
Falko Diebel, ${ }^{1, a)}$ Daniel Leykam, ${ }^{2}$ Martin Boguslawski, ${ }^{1}$ Patrick Rose,${ }^{1}$ Cornelia Denz, ${ }^{1}$ \\ and Anton S. Desyatnikov ${ }^{2}$ \\ ${ }^{1}$ Institut für Angewandte Physik and Center for Nonlinear Science (CeNoS), Westfälische Wilhelms-Universität \\ Münster, 48149 Münster, Germany \\ ${ }^{2}$ Nonlinear Physics Centre, Research School of Physics and Engineering, The Australian National University, \\ Canberra ACT 0200, Australia
}

(Received 23 April 2014; accepted 20 June 2014; published online 2 July 2014; publisher error corrected 3 July 2014)

\begin{abstract}
We experimentally demonstrate all-optical vortex switching in nonlinear coupled waveguide arrays optically induced in photorefractive media. Our technique is based on multiplexing of nondiffracting Bessel beams to induce various types of waveguide configurations. Using double- and quadruple-well potentials, we demonstrate precise control over the coupling strength between waveguides, the linear and nonlinear dynamics and symmetry-breaking bifurcations of guided light, and a power-controlled optical vortex switch. (C) 2014 AIP Publishing LLC.
\end{abstract}

[http://dx.doi.org/10.1063/1.4886414]

Low dimensional photonic structures, such as waveguide couplers, form basic building blocks for integrated optical devices and their nonlinear counterparts support the alloptical switching and routing controlled by the signal power. ${ }^{1}$ Periodic arrays of coupled waveguides represent two-dimensional photonic crystals ${ }^{2}$ and many recent fundamental results were achieved in these so-called photonic lattices. ${ }^{3,4}$ Existing fabrication techniques include etching of ridge waveguides ${ }^{5,6}$ and direct femtosecond laser writing, ${ }^{7-11}$ also supporting three-dimensional architectures. ${ }^{12,13}$ However, such waveguides are written permanently and very high peak powers up to $1000 \mathrm{~kW}$ are required to observe nonlinear effects such as discrete soliton formation. ${ }^{6,8,9}$

The optical modulation of the refractive index in photorefractive materials is an alternative approach without the above restrictions. It allows for comprehensive reconfigurability, soliton formation with low power cw laser beams, and additional electrical tunability. ${ }^{14,15}$ Optically induced periodic lattices have provided a fruitful setting for exploring nonlinear phenomena in one $\mathrm{e}^{16,17}$ and two spatial dimensions, ${ }^{18-20}$ including optical pattern formation ${ }^{21-23}$ and various analogies to quantum ${ }^{24}$ and condensed matter phenomena such as Bloch oscillations ${ }^{25}$ and Anderson localization. ${ }^{26}$ The noise-free processing of optical signals encoded in the quantized topological charges of optical vorti$\operatorname{ces}^{27,28}$ is of particular interest and has found numerous applications in nonlinear optics, ${ }^{29}$ optical micromanipulation, ${ }^{30}$ free-space data transfer, ${ }^{31}$ and quantum informatics. ${ }^{32}$ Thereby, photonic lattices well support discrete vortex ${ }^{33,34}$ and multivortex solitons, ${ }^{35,36}$ as well as power-controlled switching of vorticity. ${ }^{37-40}$

On the other hand, studies of phenomena associated with localized waveguiding structures so far have been limited to lattice defects ${ }^{41,42}$ or surfaces ${ }^{43}$ because optical induction requires nondiffracting beams, whereas spatially localized writing beams inherently diffract during propagation in homogeneous media. We solve this problem by

\footnotetext{
${ }^{\text {a) }}$ Author to whom correspondence should be addressed. Electronic mail: falko.diebel@uni-muenster.de
}

taking advantage of a recently demonstrated experimental method that allows for optical induction of arbitrary twodimensional waveguide arrays. ${ }^{44}$ This approach is based on nondiffracting Bessel beams, which are well-suited beams to cope with this unavoidable fundamental diffraction effect. The zero-order Bessel beam, for example, exhibits a transverse intensity distribution with a well confined region of high intensity which propagates in longitudinal direction without changing its shape. Therefore, the Bessel beam is suitable to induce single isolated waveguides ${ }^{45}$ but interference limits the types of multi-waveguide systems accessible via a coherent superposition. For example, higher order Bessel beams can induce ring arrays of waveguides, ${ }^{46}$ but they do not allow for individual control of coupling constants or waveguide depths. A way around this restriction is to use an incoherent superposition of multiple Bessel beams, e.g., using optical multiplexing techniques. ${ }^{44,47,48}$ The basic idea is to subsequently illuminate the photorefractive material with the individual Bessel beams at different transverse positions for a short time compared to the dielectric response time of the material. Repeating this sequence multiple times leads to a steady-state induced refractive index modulation which replicates the predetermined pattern of all waveguides. With this innovative approach, a much wider variety of potential landscapes becomes accessible to optical induction, with practically arbitrary number, positions, widths, and depths of induced waveguides.

In this Letter, we experimentally demonstrate how the multiplexing of nondiffracting Bessel beams can be harnessed to optically induce reconfigurable clusters of coupled waveguides to study propagation dynamics in lowdimensional nonlinear systems. Starting with the twowaveguide directional coupler, replicating a fundamental double-well potential, we first demonstrate selective control over all system parameters, e.g., coupling strengths and well depths, and nonlinear effects such as symmetry-breaking bifurcations. ${ }^{49,50}$ We show that this approach readily generalizes to different genuinely two-dimensional structures, such as circular waveguide arrays, ${ }^{40}$ which support a much richer variety of states and nonlinear dynamics. ${ }^{51-53}$ In this 
framework, we investigate the propagation dynamics of discrete vortices, including power-controlled switching of their vorticity, i.e., the sign of topological charge. We emphasize that our observations can be explained by relatively simple coupled mode theory and substantiate our experimental results by numerical simulations in the anisotropic photorefractive model. ${ }^{54}$

Experimentally, we use the setup sketched in Fig. 1. Light from a frequency-doubled $\mathrm{Nd}: \mathrm{YVO}_{4}$ laser at a wavelength $\lambda=532 \mathrm{~nm}$ is divided into two beams, each illuminating a high-resolution programmable phase-only LCoS spatial light modulator (SLM). Encoding a pre-calculated phase map onto the modulator allows us to simultaneously shape the intensity and phase distribution in order to produce the desired complex light field. The first modulator (SLM1) thereby generates a nondiffracting induction beam, while the second (SLM2) structures the probe beam. The power of each inducing Bessel beam is set to $P_{\text {Bessel }} \approx 1.3 \mu \mathrm{W}$. In all experiments, we use a $20 \mathrm{~mm}$ long photorefractive strontium barium niobate $(\mathrm{SBN})$ crystal which is externally biased with a dc electric field $E_{\text {ext }}=2 \mathrm{kV} / \mathrm{cm}$ applied along the optical caxis to generate focusing nonlinearity. To erase an inscribed refractive index modulation, we can illuminate the crystal with white light for few minutes. Thus, this setup provides a highly flexible platform to reversibly realize various nonlinear refractive index landscapes. We record the intensity and phase distributions at the front and back face of the crystal with a CCD camera mounted on a translation stage. For phase measurements, we interfere the probe with an additional reference beam.

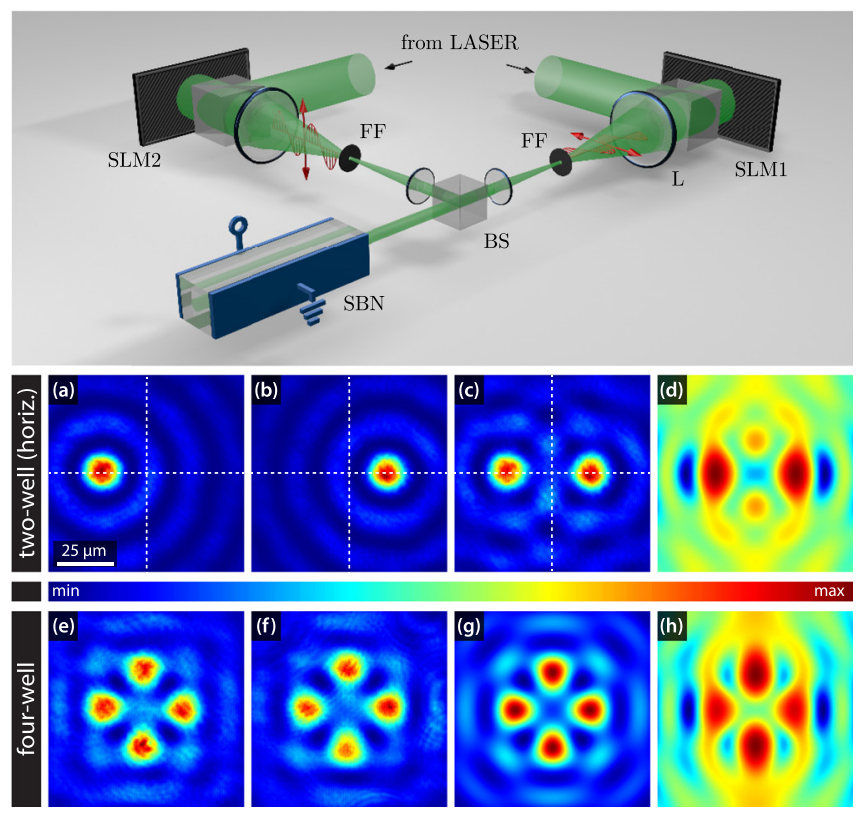

FIG. 1. Top panel: experimental setup. BS: beam splitter, FF: Fourier filter, L: lens, SLM: spatial light modulator. Bottom panels: Incremental multiplexing of multi-well structures with Bessel beams. (a)-(d) Horizontal twowell structure: (a) and (b) experimentally measured intensity of induction beams, (c) effective induction beam intensity as digital superposition of these beams, and (d) the simulated refractive index profile for this configuration. (e)-(h) Four-well structure: (e) and (f) total effective intensity of the induction beam at the front and back crystal faces, and $(\mathrm{g})$ and $(\mathrm{h})$ simulated intensity and refractive index.
First, to demonstrate our technique, we consider the simplest nonlinear two-waveguide configuration, ${ }^{1}$ generated by incrementally multiplexing two zero-order nondiffracting Bessel beams. In Figs. 1(a)-1(c), the experimentally recorded intensities for each Bessel beam and the intensity of the incoherent superposition are displayed. The numerically calculated refractive index modulation based on the anisotropic photorefractive model for the corresponding experimental parameters is shown in Fig. 1(d) (see supplemental material for details). There are two principal orientations of the structure: parallel or perpendicular to the c-axis, which produce different refractive index profiles due to the anisotropic material response. ${ }^{54}$ We use the former one which generates a configuration of two well-defined waveguides. Their depths can be controlled independently through the Bessel beam intensities or the relative exposure times.

To first approximation, each potential well hosts a single bound mode $\psi$ with an exponentially decaying tail, $\psi(r) \sim \exp (-\sqrt{\beta} r)$, where $\beta$ is the mode's propagation constant and $r$ is the distance from the well center. Within the coupled mode approach, the coupling strength between the wells scales with their separation $d$ as $C(d) \propto \exp \left(-d / d_{0}\right)$. Thus, the coupling strength critically depends on the waveguide separation, which we can control by changing the distance between the two Bessel beams. The evanescent coupling between the wells results in mode hybridization into symmetric and antisymmetric linear combinations $\psi_{ \pm}$ with propagation constants $\beta_{ \pm}=\beta \pm C$. An excitation of a single well always excites superposition of both modes $\psi_{ \pm}$, resulting in oscillations of mode intensities in each waveguide $I_{1}$ and $I_{2}$. In the linear regime at low probe beam power, this oscillation evolves as $I_{1}(z) \propto \cos ^{2}(C z)$ and $I_{2}(z) \propto \sin ^{2}(C z)$. Since, in our experiments, the total propagation distance is fixed by the length of the crystal $L=20 \mathrm{~mm}$, the coupling strength $C$ controls the relative output intensities.

In Fig. 2, we demonstrate the control over the coupling constant by observing the splitting of the output power between the two waveguides at the back face of the crystal. We select four particular values for $d$ to experimentally present the extreme points of the oscillation. For $d=36 \mu \mathrm{m}$ in Fig. 2(a), the coupling is too weak, so no oscillation is observed. Decreasing the distance $d$, we increase the coupling constant $C$ and observe equal outputs in Fig. 2(b) with $d=24 \mu \mathrm{m}$, full transfer of power from right to left waveguide in Fig. 2(c) with $d=20 \mu \mathrm{m}$, and full cycle of the intensity oscillation at the given crystal length in Fig. 2(d) with $d=14 \mu \mathrm{m}$ which allows us to estimate $C \approx \pi / L$ in this case.

Repeating the experiment for a whole set of well separations $d$, we measure the output intensities in the two wells $I_{1,2}$ to extract the variation of the coupling strength $C(d)$. The results are plotted in Fig. 3. They reveal a good agreement between our experiments and the predictions from the coupled mode theory. Thus, observing intensity oscillations allows us to precisely calibrate our setup in order to adjust the coupling strength.

Increasing the probe beam power, we enter the nonlinear regime. The coupled mode theory for a nonlinear dimer is an integrable model, ${ }^{1}$ forming a simple setting for exploring 

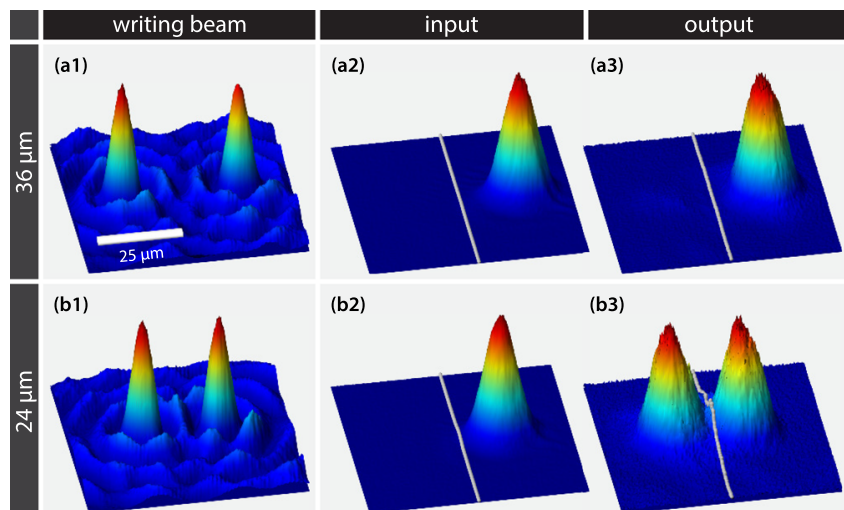

(b3)

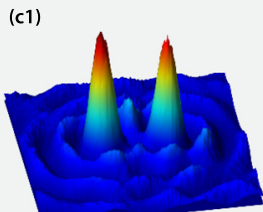

(c2)

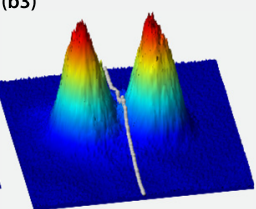

(c3)
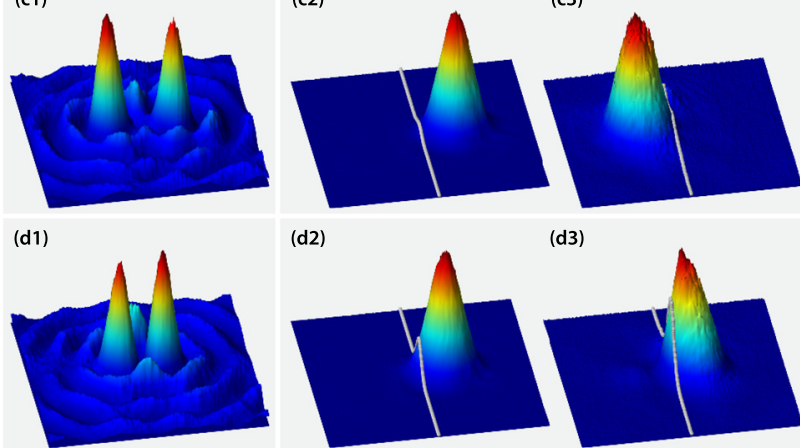

(d2)

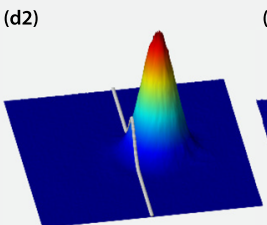

(d3)

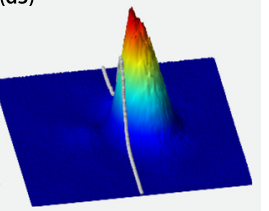

FIG. 2. Oscillations of normalized output intensities in a linear two-well photonic potential for different well separations ((a)-(d)). (Left) Digital superpositions of the individually measured intensity distributions of the Bessel beams at the front face, (middle) probe beam intensity at the front face, and (right) probe beam at the back face.

interplay between linear oscillations and nonlinear selftrapping. The nonlinear modes of the dimer are shown in Fig. 3(b). A symmetry-breaking pitchfork bifurcation occurs
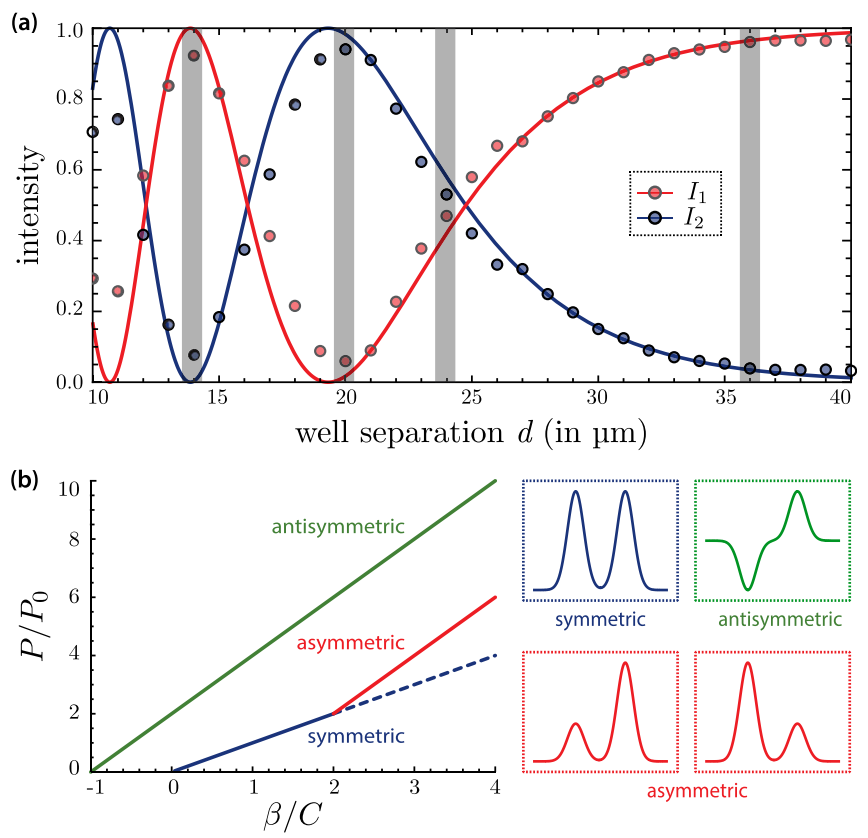

FIG. 3. Coupled mode theory and symmetry breaking bifurcation. (a) Measurements of the normalized intensities $I_{1}$ and $I_{2}$ as a function of well separation $d$, the shaded areas correspond to the measurements shown in Fig. 2. The solid lines represent the predictions from coupled mode theory. (b) Modes of the nonlinear dimer and their linear stability: solid lines indicate stable modes and dashed line shows the unstable mode.

at a critical power $P_{\mathrm{c}}=2 .{ }^{50}$ Above this power, the symmetric excitation of both waveguides becomes unstable, resulting in a localization of the output in one single waveguide (asymmetric mode). In this case, the output intensity is highly sensitive to small perturbations. The nonlinear antisymmetric mode, in contrast, remains stable against perturbations, with both wells remaining strongly excited at the output.

In Fig. 4, the experimental results for the symmetrybreaking bifurcation are shown for a waveguide distance of $d=20 \mu \mathrm{m}$, confirming that we are in the nonlinear regime, $P>P_{\mathrm{c}}$. If any small asymmetry is introduced to the waveguides, for example, by changing the relative power of the two Bessel beams, or simply due to experimental noise, the intensity of the symmetric mode is easily directed to the stronger waveguide at the output, see Fig. 4(d2). The intensity profile is slightly shifted towards the center. This could be explained by the relatively low probe beam intensity in the vicinity of the bifurcation point where the nonlinearity is not sufficiently strong to completely trap all intensity in one well. We conclude that the coupled mode approximation remains valid also when the probe beam is strong enough to observe nonlinear effects.

The optical induction technique is not limited to such simple systems like the directional coupler introduced above and readily generalizes to larger arrays of coupled waveguides. With more than two waveguides, the coupled mode equations are no longer integrable, allowing for a much richer variety of nonlinear dynamics, including chaos. ${ }^{53}$ Ring-like configurations of waveguides are particularly interesting because they support modes with energy circulation, e.g., discrete vortices, ${ }^{33,34}$ which have no one-dimensional counterparts. ${ }^{40}$ We here specifically aim for the implementation of a stable switching, but extensions towards complex nonlinear dynamics are possible since our system provides a lot of flexibility with controlling the photonic structure and shaping the probe beam to exciting different types of oscillations.

To explore the dynamics of a discrete optical vortex, we employ a basic configuration of four waveguides shown in Figs. 1(e)-1(h). For simplicity, we consider four identical Bessel beams for the optical induction. Due to the anisotropic response of the photorefractive SBN, this does not produce four identical waveguides. Instead, there already is a detuning $\delta_{\mathrm{L}}$ between the depths of the horizontally and
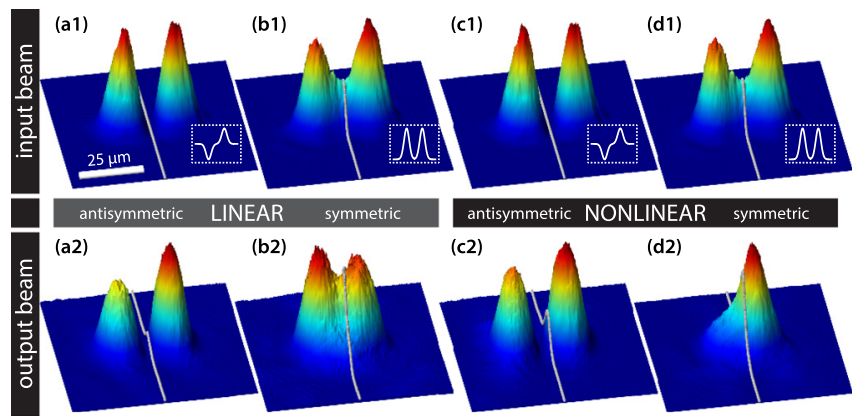

FIG. 4. Nonlinear symmetry breaking in two-well potential. (Top row) Probe beam at the front face, (bottom row) output at the back face. (a) and (b) Linear (anti)symmetric modes, (c) and (d) nonlinear (anti)symmetric modes. 
vertically aligned waveguide pairs. However, the symmetry of the induction beam ensures that opposite waveguides are identical. This system hosts a pair of double-well antisymmetric eigenmodes, $\{|H\rangle,|V\rangle\}$, aligned either horizontally $(\mathrm{H})$ or vertically $(\mathrm{V})$ and consisting of a $\pi$-out-of-phase excitations of opposite waveguides in each pair. These modes persist even when nonlinearity is included and they are split by a detuning $\delta=\delta_{\mathrm{L}}+\delta_{\mathrm{NL}}$, which incorporates linear splitting of the waveguide depths $\delta_{\mathrm{L}}$ and nonlinear shift of their propagation constants $\delta_{\mathrm{NL}}$, proportional to the strength of the mode excitation.

A discrete vortex is an excitation with the intensity localized at the positions of the waveguides and a phase winding around the origin. ${ }^{40}$ For a discrete vortex in a fourwell system, the phase reads as $\phi_{n}=m 2 \pi n / 4$, where $m$ is the topological charge and $n=1, \ldots, 4$ specifies the waveguide. The trivial case with $m=0$ shows no phase circulation, while $m= \pm 1$ corresponds to vortices with (anti-) clockwise circulation of energy. In this case, opposite vortex lobes are $\pi$ out of phase and thus the discrete vortex can be written as superposition of the two antisymmetric eigenmodes, $|H\rangle \pm \mathrm{i} \eta|V\rangle$, where $\eta$ is a relative strength of excitation. For the case of zero detuning, $\delta=0$, the two eigenmodes $|H\rangle$ and $|V\rangle$ are degenerate, following that vortices as their superpositions also are eigenmodes of the system. In contrast, if $\delta \neq 0$, the modes $|H\rangle$ and $|V\rangle$ accumulate a phase difference during propagation, which turns the vortex first into a multipole state and then into an oppositely charged vortex. This periodic oscillation of the topological charge of discrete vortices was originally predicted theoretically for discrete vortices in square periodic lattices. ${ }^{37}$ Corresponding experiments revealed complex dynamics with multiple phase dislocations and charge-dependent deformations of the intensity of selftrapped states. $^{38,39}$

Here, we demonstrate that charge flipping of discrete optical vortices can also be observed in finite systems of few coupled waveguides. In contrast to spatially extended lattices, our system does not show discrete diffraction and thus the discrete vortex does not suffer spreading into the lattice at low power levels. The probe beam completely stays localized only due to the presence of the coupled waveguide structure. Therefore, we can implement a power-controlled vortex switch where the output state purely depends on the probe beam power. We have tuned the well depths of our system, shown in Figs. 1(e)-1(h), such that the linear splitting is $\delta_{\mathrm{L}} \mathrm{L} \approx \pi$. Hence, a linear, left-handed vortex input has its topological charge inverted after propagating through the crystal, as experimentally shown in Figs. 5(a) and 5(b).

Entering the nonlinear regime, the relative strength $\eta$ now determines the nonlinear detuning $\delta_{\mathrm{NL}}$, which can be adjusted to compensate the linear detuning. When $\eta \neq 1$, the two eigenmodes will experience different nonlinear shifts to their propagation constants. By making the shallower pair of wells host the brighter lobes of the probe beam, increasing the probe beam power will decrease the effective detuning $\delta$. By increasing the probe beam power, we can continuously tune the output phase profile, while simultaneously preserving the intensity pattern, as shown in Fig. 5 and more detailed here. ${ }^{55}$ At a critical vortex beam power of $P_{\text {vortex }} \approx 102 \mathrm{nW}$, the nonlinear phase shift $\delta_{\mathrm{NL}}$ exactly

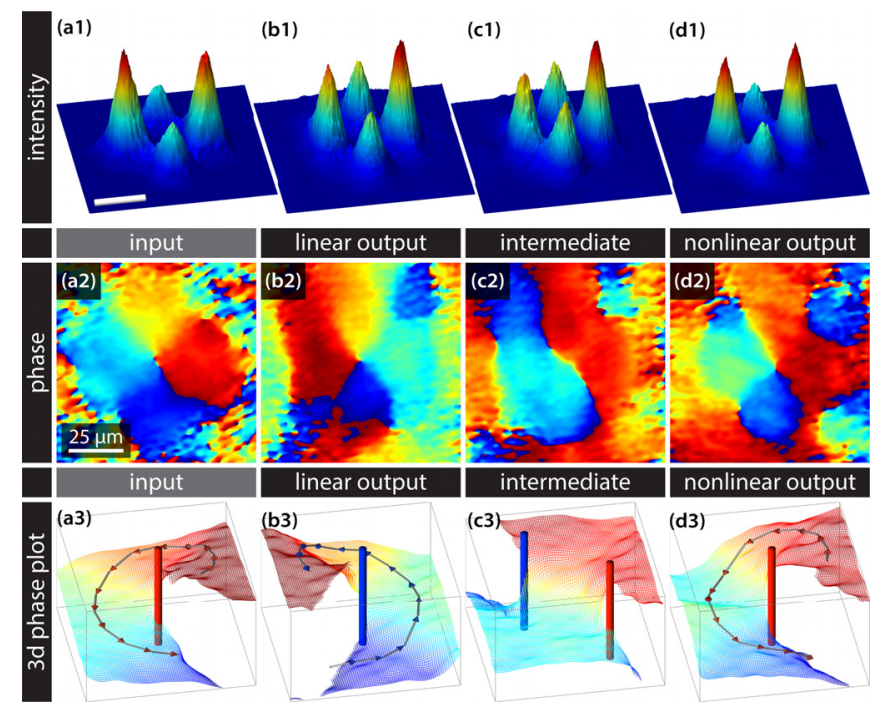

FIG. 5. Nonlinear vortex switch in four coupled waveguides. (a) Intensity and phase profile of the input beam at the front face. Intensity and phase at the back face for (b) linear propagation, (c) for an intermediate power level, (d) for the certain power level at which the input vortex is exactly preserved.

balances the effect of the well detuning, such that $\delta=0$ and the input vortex is completely preserved (Fig. 5(d)) and the nonlinear eigenmodes $\{|H\rangle,|V\rangle\}$ are stable up to small modulation due to experimental noise. Therefore, just by changing the input power, we can control the charge of the vortex at the output, or even select an intermediate vortexantivortex pair. This demonstrates an all-optical switch of the vortex topological charge. ${ }^{40}$

In summary, we have demonstrated the experimental realization of an all-optical vortex switch which allows us to control the vorticity by simply changing the beam power. The two-dimensional array of coupled nonlinear waveguides was optically induced with an innovative approach based on multiplexing of Bessel beams. Both one- and twodimensional geometries with individually positioned and controlled waveguides are now accessible to optical induction. Moreover, we have demonstrated precise control over the coupling strength between the waveguides and nonlinear symmetry-breaking bifurcations, which, as we showed, is well described by the coupled mode theory. The presented approach may be further generalized to other types of propagation-invariant or self-similar beams, such as selfaccelerating Bessel-like beams, ${ }^{56}$ thus providing a versatile tool to explore nonlinear dynamics in low-dimensional systems.

This work was supported by the German-Australian DAAD-Go8 travel grant and the Australian Research Council.

${ }^{1}$ S. M. Jensen, IEEE J. Quantum Electron. 18, 1580 (1982).

${ }^{2}$ J. D. Joannopoulos, S. G. Johnson, J. N. Winn, and R. D. Meade, Photonic Crystals: Molding the Flow of Light, 2nd ed. (Princeton University Press, 2008).

${ }^{3}$ D. N. Christodoulides, F. Lederer, and Y. Silberberg, Nature 424, 817 (2003).

${ }^{4}$ F. Lederer, G. I. Stegeman, D. N. Christodoulides, G. Assanto, M. Segev, and Y. Silberberg, Phys. Rep. 463, 1 (2008).

${ }^{5}$ P. Millar, J. S. Aitchison, J. U. Kang, G. I. Stegeman, A. Villeneuve, G. T. Kennedy, and W. Sibbett, J. Opt. Soc. Am. B 14, 3224 (1997). 
${ }^{6}$ H. S. Eisenberg, Y. Silberberg, R. Morandotti, A. R. Boyd, and J. S. Aitchison, Phys. Rev. Lett. 81, 3383 (1998).

${ }^{7}$ K. M. Davis, K. Miura, N. Sugimoto, and K. Hirao, Opt. Lett. 21, 1729 (1996).

${ }^{8}$ A. Szameit, D. Blömer, J. Burghoff, T. Schreiber, T. Pertsch, S. Nolte, A. Tünnermann, and F. Lederer, Opt. Express 13, 10552 (2005).

${ }^{9}$ A. Szameit, J. Burghoff, T. Pertsch, S. Nolte, A. Tünnermann, and F. Lederer, Opt. Express 14, 6055 (2006).

${ }^{10}$ A. Szameit and S. Nolte, J. Phys. B: At. Mol. Opt. Phys 43, 163001 (2010).

${ }^{11}$ R. Osellame, G. Cerullo, and R. Ramponi, "Femtosecond laser micromachining," Topics in Applied Physics, Vol. 123 (Springer, Berlin, 2012).

${ }^{12}$ R. Keil, M. Heinrich, F. Dreisow, T. Pertsch, A. Tünnermann, S. Nolte, D. N. Christodoulides, and A. Szameit, Sci. Rep. 1, 94 (2011).

${ }^{13}$ M. C. Rechtsman, J. M. Zeuner, Y. Plotnik, Y. Lumer, D. Podolsky, F. Dreisow, S. Nolte, M. Segev, and A. Szameit, Nature 496, 196 (2013).

${ }^{14}$ N. K. Efremidis, S. Sears, D. N. Christodoulides, J. W. Fleischer, and M. Segev, Phys. Rev. E 66, 046602 (2002).

${ }^{15}$ J. W. Fleischer, G. Bartal, O. Cohen, T. Schwartz, O. Manela, B. Freedman, M. Segev, H. Buljan, and N. K. Efremidis, Opt. Express 13, 1780 (2005).

${ }^{16} \mathrm{~J}$. W. Fleischer, T. Carmon, M. Segev, N. K. Efremidis, and D. N. Christodoulides, Phys. Rev. Lett. 90, 023902 (2003).

${ }^{17}$ D. N. Neshev, E. Ostrovskaya, Y. S. Kivshar, and W. Krolikowski, Opt. Lett. 28, 710 (2003).

${ }^{18}$ J. W. Fleischer, M. Segev, N. K. Efremidis, and D. N. Christodoulides, Nature 422, 147 (2003).

${ }^{19}$ B. Terhalle, A. S. Desyatnikov, C. Bersch, D. Träger, L. Tang, J. Imbrock, Y. S. Kivshar, and C. Denz, Appl. Phys. B 86, 399 (2007).

${ }^{20}$ P. Rose, T. Richter, B. Terhalle, J. Imbrock, F. Kaiser, and C. Denz, Appl. Phys. B 89, 521 (2007).

${ }^{21}$ D. Gomila, R. Zambrini, and G.-L. Oppo, Phys. Rev. Lett. 92, 253904 (2004).

${ }^{22}$ N. Marsal, D. Wolfersberger, M. Sciamanna, G. Montemezzani, and D. N. Neshev, Opt. Lett. 33, 2509 (2008).

${ }^{23}$ A. A. Sukhorukov, N. Marsal, A. Minovich, D. Wolfersberger, M. Sciamanna, G. Montemezzani, and D. N. Neshev, Opt. Lett. 35, 3568 (2010).

${ }^{24}$ S. Longhi, Laser Photonics Rev. 3, 243 (2009).

${ }^{25}$ H. Trompeter, W. Krolikowski, D. Neshev, A. S. Desyatnikov, A. A. Sukhorukov, Y. S. Kivshar, T. Pertsch, U. Peschel, and F. Lederer, Phys. Rev. Lett. 96, 053903 (2006).

${ }^{26}$ T. Schwartz, G. Bartal, S. Fishman, and M. Segev, Nature 446, 52 (2007).

${ }^{27}$ M. S. Soskin and M. V. Vasnetsov, Prog. Opt. 42, 219 (2001).

${ }^{28}$ M. R. Dennis, K. O'Holleran, and M. J. Padgett, Prog. Opt. 53, 293 (2009).

${ }^{29}$ A. S. Desyatnikov, Y. S. Kivshar, and L. Torner, Prog. Opt. 47, 291 (2005).

${ }^{30}$ M. Padgett and R. Bowman, Nat. Photonics 5, 343 (2011).
${ }^{31}$ J. Wang, J.-Y. Yang, I. M. Fazal, N. Ahmed, Y. Yan, H. Huang, Y. Ren, Y. Yue, S. Dolinar, M. Tur, and A. E. Willner, Nat. Photonics 6, 488 (2012).

${ }^{32}$ A. Mair, A. Vaziri, G. Weihs, and A. Zeilinger, Nature 412, 313 (2001).

${ }^{33}$ D. N. Neshev, T. J. Alexander, E. A. Ostrovskaya, Y. S. Kivshar, H. Martin, I. Makasyuk, and Z. Chen, Phys. Rev. Lett. 92, 123903 (2004).

${ }^{34}$ J. W. Fleischer, G. Bartal, O. Cohen, O. Manela, M. Segev, J. Hudock, and D. N. Christodoulides, Phys. Rev. Lett. 92, 123904 (2004).

${ }^{35}$ B. Terhalle, T. Richter, A. S. Desyatnikov, D. N. Neshev, W. Krolikowski, F. Kaiser, C. Denz, and Y. S. Kivshar, Phys. Rev. Lett. 101, 013903 (2008).

${ }^{36}$ B. Terhalle, T. Richter, K. J. H. Law, D. Göries, P. Rose, T. J. Alexander, P. G. Kevrekidis, A. S. Desyatnikov, W. Krolikowski, F. Kaiser, C. Denz, and Y. S. Kivshar, Phys. Rev. A 79, 043821 (2009).

${ }^{37}$ T. J. Alexander, A. A. Sukhorukov, and Y. S. Kivshar, Phys. Rev. Lett. 93, 063901 (2004).

${ }^{38}$ A. Bezryadina, D. N. Neshev, A. S. Desyatnikov, J. Young, Z. Chen, and Y. S. Kivshar, Opt. Express 14, 8317 (2006).

${ }^{39}$ A. Bezryadina, E. Eugenieva, and Z. Chen, Opt. Lett. 31, 2456 (2006).

${ }^{40}$ A. S. Desyatnikov, M. R. Dennis, and A. Ferrando, Phys. Rev. A 83, 063822 (2011).

${ }^{41}$ I. Makasyuk, Z. Chen, and J. Yang, Phys. Rev. Lett. 96, 223903 (2006).

${ }^{42}$ B. Freedman, G. Bartal, M. Segev, R. Lifshitz, D. N. Christodoulides, and J. W. Fleischer, Nature 440, 1166 (2006).

${ }^{43}$ X. Wang, A. Bezryadina, Z. Chen, K. G. Makris, D. N. Christodoulides, and G. I. Stegeman, Phys. Rev. Lett. 98, 123903 (2007).

${ }^{44}$ F. Diebel, P. Rose, M. Boguslawski, and C. Denz, Appl. Phys. Lett. 104, 191101 (2014).

${ }^{45}$ X. Wang, Z. Chen, and P. G. Kevrekidis, Phys. Rev. Lett. 96, 083904 (2006).

${ }^{46}$ R. Fischer, D. N. Neshev, S. López-Aguayo, A. S. Desyatnikov, A. A. Sukhorukov, W. Krolikowski, and Y. S. Kivshar, J. Mater. Sci. Mater. Electron. 18, 277 (2007).

${ }^{47}$ P. Rose, B. Terhalle, J. Imbrock, and C. Denz, J. Phys. D: Appl. Phys. 41, 224004 (2008).

${ }^{48}$ M. Boguslawski, A. Kelberer, P. Rose, and C. Denz, Opt. Express 20, 27331 (2012).

${ }^{49}$ P. G. Kevrekidis, Z. Chen, B. A. Malomed, D. J. Frantzeskakis, and M. I. Weinstein, Phys. Lett. A 340, 275 (2005).

${ }^{50}$ G. Theocharis, P. G. Kevrekidis, D. J. Frantzeskakis, and P. Schmelcher, Phys. Rev. E 74, 056608 (2006).

${ }^{51}$ T. Kapitula, P. G. Kevrekidis, and Z. Chen, SIAM J. Appl. Dyn. Syst. 5, 598 (2006).

${ }^{52}$ C. Wang, G. Theocharis, P. G. Kevrekidis, N. Whitaker, K. J. H. Law, D. J. Frantzeskakis, and B. A. Malomed, Phys. Rev. E 80, 046611 (2009).

${ }^{53}$ T. J. Alexander, D. Yan, and P. G. Kevrekidis, Phys. Rev. E 88, 062908 (2013).

${ }^{54}$ A. A. Zozulya and D. Z. Anderson, Phys. Rev. A 51, 1520 (1995).

${ }^{55}$ See supplemental material at http://dx.doi.org/10.1063/1.4886414 for more experimental and numerical details.

${ }^{56}$ J. Zhao, P. Zhang, D. Deng, J. Liu, Y. Gao, I. D. Chremmos, N. K. Efremidis, D. N. Christodoulides, and Z. Chen, Opt. Lett. 38, 498 (2013). 